\title{
The Thing we should not Forget in Brachytherapy: Inverse Square Law
}

\author{
(1) Görkem TÜRKKAN,' (D) Alaattin ÖZEN² \\ 'Department of Radiation Oncology, Muğla Sıtkı Koçman University Faculty of Medicine, Muğla-Turkey \\ ${ }^{2}$ Department of Radiation Oncology, Eskişehir Osmangazi University Faculty of Medicine, Eskişehir-Turkey
}

\section{Dear Editor,}

We read the article entitled "The Effects of the Amount of Bladder Filling on Normal Tissue Doses in 3-dimensional HDR Vaginal Cuff Brachy Therapy" by Er et al.[1] with great interest. After congratulating the authors for this insightful work, we would like to make a few constructive criticisms and contributions.

In this study, dose-volume parameters (DVPs) of organs at risk (OAR) (volume receiving $50 \%$ of the dose (V50\%), dose received by $50 \%$ of the volume (D50\%), the minimum dose for the $2 \mathrm{~cm}^{3}$ volume receiving the highest dose (D2cc) for bladder; D2cc for rectum and sigmoid; D50\% and D2cc for bowel) were compared in the case of bladder filling (BF) of $50 \mathrm{cc}$ and $150 \mathrm{cc}$. For this purpose, CT-scan was performed at two different amounts of BF (50 and $150 \mathrm{cc}$ ), and $150 \mathrm{cc}$ filling was preferred in the presence of a significant visual difference in removal of the bowels away from the applicator. After placement, the applicator was immobilized by attaching it to a rigid external fixator on the BRT bed. Proximal $1 / 3$ vagina was treated in all patients. HDR BRT dose was administered, and the Ir-192 source had been prescribed to $0.5 \mathrm{~cm}$ depth from the vaginal mucosa (SA surface). A total dose (TD) of 18 Gy was performed with a fraction dose of $6 \mathrm{~Gy}$ administered once or twice a week.

When the data of eight patients included in their study were reviewed, although the same applicator is used and the same prescribed dose is defined at $5 \mathrm{~mm}$ in depth in the same way, the differences between obtained target doses are seen in Table 2. However, the possible reason for this difference and whether it is statistically significant or not is not mentioned in the study. We should note that the doses measured at a certain distance from the source will be different if this issue is due to different Dwell times rather than contouring. In addition to this, it is given in Table 3 that the $\mathrm{D} 2 \mathrm{cc}$ value increased and the D50\% value decreased statistically when we filled the bladder more. Both these two values were using to define the dose received by a certain volume, and the distance of these volumes from the source was not evaluated in this study.

In conclusion, in the treatment of brachytherapy, the most important thing in determining how much dose will occur on a point or in a volume is the distance of this point or volume from the source. To our knowledge up to now, of the four factors (distance-inverse square law, absorption and scattering in the source core and encapsulation, photon attenuation, scattering in the surrounding medium) may influence the dose distribution in brachytherapy, an inverse-square law is by far the most important.[2] The direction of the bladder expansion due to bladder filling will have a determining influence on bladder D2cc or D50\% values, whether positively or negatively.

\section{References}

1. Er I, Kınay S, Kandemir R, Obuz F, Demiral AN. The Effects of the Amount of Bladder Filling on Normal Tissue Doses in 3-dimensional HDR Vaginal Cuff Brachy Therapy. Turk J Oncol 2019;34(4):275-82.

2. Williamson JF, Brenner DJ. Physics and Biology of Brachytherapy. In: Halperin EC, Wazer DE, Perez CA, Brady LW, editors. Perez \& Brady's Principles and Practice of Radiation Oncology. 7th ed. Philadelphia: Wolters Kluwer Health; 2018. p. 530-81. 